\title{
Review of: "Out-of-hospital cardiac arrest and ambient air pollution: A dose-effect relationship and an association with OHCA incidence"
}

José Brito

Potential competing interests: The author(s) declared that no potential competing interests exist.

The authors purported to assess the impact of short-term exposure to various health pollutants on the incidence of Out-of-hospital cardiac arrest (OHCA) in the Po Valley in Northern Italy, and to establish a dose-effect relationship which could help predicting OHCA incidence. The main findings of this study were that all pollutant concentrations were strongly associated with high incidence of OCHA, even after correction for relevant meteorological variables, such as temperature and relative humidity. Based on these findings, the authors conclude that their results clarify the link between pollutants and the acute risk of cardiac arrest. There are, however, several issues affecting the methodologies of data analysis used in this study that put the robustness and validity of its conclusions in jeopardy, as detailed next.

1. A discussion of the criteria used to check the validity of the ambient air data registered in the monitoring stations included in this work is missing. It does not suffice to say that the data were available from the regional agency for environmental protection (ARPA). As the Directive 2008/50/EC of the European Parliament and of the Council, of 21 May 2008, clearly establishes, there is a minimum percentage of valid data required for regulatory and research purposes. Therefore, this important aspect for the robustness of the conclusions requires further information and clarification.

2. In the absence of further discussion and explanation, for example through modelling of the pollutant dispersion within a given region based on actual data, the true exposure to pollutants considered in this study in each province may be misrepresented. As suggested by Figure 1, a large discrepancy between the number and location of the monitoring stations and the OCHA location is conceivable. Therefore, further information is required to identify clusters of OCHA locations that fall within the type of environment and emission influence of each station, which helps clarifying exposure representativeness minimally.

3. Information on criteria for classifying the local sampling points (for example, type of station, representativeness, and type of influence) would be helpful. Since it is conceivable that different monitoring stations may be of different type (urban, suburban or rural) and influence (background, industry or traffic) a clear explanation and justification must be provided in case averages of atmospheric levels of a given pollutant were computed among stations of different types.

4. Other relevant information that is missing is on how PM2.5 concentrations were estimated for the 14 stations that did not measure this pollutant. There are no comments on this important issue, as the 
relationship between PM2.5 and morbidity and mortality is much stronger than that of PM10.

5. The authors have corrected their models for the effect of relevant meteorological covariates, such as temperature and relative humidity, but only in the single pollutant scenario. However, a more realistic scenario is actual exposures to mixtures of air pollutants, as those mixtures have proven to be more toxic than the individual compounds, as is the case of $\mathrm{SO} 2+\mathrm{O} 3, \mathrm{PM} 10+\mathrm{O} 3$ and $\mathrm{NO} 2+\mathrm{O} 3$ (please see, Williams, M.L., Atkinson, R.W., Anderson, H.R., Kelly, F.J., 2014. Associations between daily mortality in London and combined oxidant capacity, ozone and nitrogen dioxide. Air Qual. Atmos. Heal. 7, 407-414. https://doi.org/10.1007/s11869-014-0249-8). In this scenario, the robustness of the conclusions of the effect of a given pollutant on the incidence of ACHA may be underestimated.

6. More than once, the counter trend detected for $\mathrm{O} 3$ is mentioned in this study, but this important outcome is never discussed by the authors. It is true that the 03 formation process is complex, involves NO2 and volatile organic compounds, is driven by sunlight, solar radiation and temperature. However, that should not preclude the authors from analyzing the trend in $\mathrm{O} 3$ observed in this work with published reports (for example, EEA Report No 12/2018, 2018. Air quality in Europe - 2018 report. Luxembourg. https://doi.org/10.2800/777411).

7. The authors should use more appropriate statistical terminology. For example, variables, categorical or continuous, are not "compared", as stated at the beginning of Statistical analysis section; at best, groups, independent or paired, are compared using variables as criteria for that comparison. Instead, what is usually assessed between variables is their association or relationship. Along this line, what is meant by the following statement in the same section: "An univariable logistic regression model was run for every pollutant to test the strength of association of the probability of having an incidence of OHCA higher than median value."? Presumably, what the authors want to do is to assess the association between exposure to a given pollutant and OHCA, using a logistic function that eventually will link the level of exposure and the probability of that event. However, that is not clear from the statement above.

8. The use of multiple correlation coefficient needs clarification. First, why is it relevant to assess the correlation between the concentration of a given pollutant between provinces? Second, and presumably, the estimate of a multiple correlation was performed using a multivariate regression model. If that was the case, which of the pollutant was considered the dependent variable in such models, and why? Given the aims of this work, it would seem more important, in the environmental and population health perspective, to compare the different provinces both for the level of atmospheric pollution and OCHA incidence, but that could not be achieved by using multivariable regression neither.

9. As a matter of fact, it is the quality of the methods of data analysis put in place in this work that compromises its conclusions seriously. First, the use of linear or logistic regression models relies on the assumption of residual independence, which is seriously compromised in long time series of data collected in the same location, as is the case in this study. In fact, a strong autocorrelation and trend are expected in both series of pollutant and OCHA data because those data were registered hourly 
and/or daily. Therefore, such correlation must be considered in the data analysis as traditional regression models are not fit for that.

10. Instead of traditional regression models, time-series analysis, or linear mixed models, which were designed precisely to address the issue of autocorrelation and heterogeneous variances between repeated measures on the same observation units, should have been used. Or at least, a convincing proof of the absence of those features in the analyzed data, and a clear demonstration that the assumption for regression models were met should have been provided. Unfortunately, the authors have failed to do that, their results may be overemphasized, and their methods should be revised. In its current state, the validity of their conclusions is undermined by these issues and weaknesses. 